\title{
Fußglieder an Käferfühlern
}

\section{(zngleich: Homoeosis bei Arthropoden V.Mritteilung).}

Von

\section{Hang Przibram.}

(Aus der Biologischen Versuchsanstalt der kaiserl. Akadennie der Wissenschaften in Wien [Zoologische Abteilung] ${ }^{1}$ ).)

Mit Tafel IV und 5 Textabbildungen.

(Eingegangen am 23. Juli 1918.)

Inhaltstibersicht. Seite

I. Einfache Regenerate an Küferfühler. . . . . . . . . . . . 52

II. Ersatzheteromorphose am Käferfühler . . . . . . . . . . . . . 54

III. Zusatzheteromorphosen am Käferfühler . . . . . . . . . . . 57

IV. Theoretische Erörterung . . . . . . . . . . . . . . . . . 61

Zusammenfassung ............... . . 66

Literaturverzeichnis . . . . . . . . . . . . . 67

Tafelerklärung . . . . . . . . . . . . . . . . 68

I. Einfache Regenerate am Käferfïhler.

In unserem entwicklungsmechanischen Nuseum befinden sich mehrere Käfer mit abnormer Fühlerausbildung, welche im Freien gefunden worden sind.

Da wir aus den Experimenten von Gadeau de Kerville (1890), Tornier (1901), Werber (1905) und Megušar (1907) wissen, daß an der Larve verstümmelte Fühler regenerieren und an der Imago in unvollständiger Reproduktion fixiert werden, wenn nicht genügend Zeit vor der Metamorphose zur Wiedererreichung des normalen Zustandes gegeben war, so liegt es nahe, die in der Natur gefundenen Fühlermonstrositäten ebenfalls als Regenerate anzusprechen.

Zunächst seien zwei Fälle von Schneekäfern (Telephorus) beschrieben, welche die gewöhnliche, vom normalen Fühler wenig abweichende Regeneratform aufweisen und uns für die Beurteilung der dann in den Abschnitten II und III zu beschreibenden stärker abweichenden Fühlermißbildungen von Nutzen sein werden.

Nr. 1. Telephorus rusticus Fallèn (Textabb. 1; Taf.IV, Abb. 1). Linker Fühler von Anfang an schmäler als der normale rechte; besteht aus den drei deutlich abgegliederten; aber zu kleinen ersten Gliedern und einer

1) Ein Auszug dieser-Arbeit erschien mit gleichlautendem Titel als Mitteilung Nr. 29 aus der Biol. Versuchsanstalt dor kais. Akad. d..Wissensch., Zool. Abt, im Sitzungsanzeiger Nr. 17, 1918. 
rundlichen Knospe, welche die Breite der normalen Fühlerglieder erreicht und an ihrem Ende die Andeutung einer weiteren Abgliederung eines Endgliedes trägt. Die Behaarung sämtlicher Glieder des linken Fühlers entspricht derjenigen des normalen rechten. : Insbesondere ist das schwach abgegliederte Fühlerende mit Endhaaren von zwei verschiedenen Längen versehen. Falls es zulässig ist, Haare nur ihrer Länge nach zu homologisieren, würde sich das 4. Glied des linken Fühlers

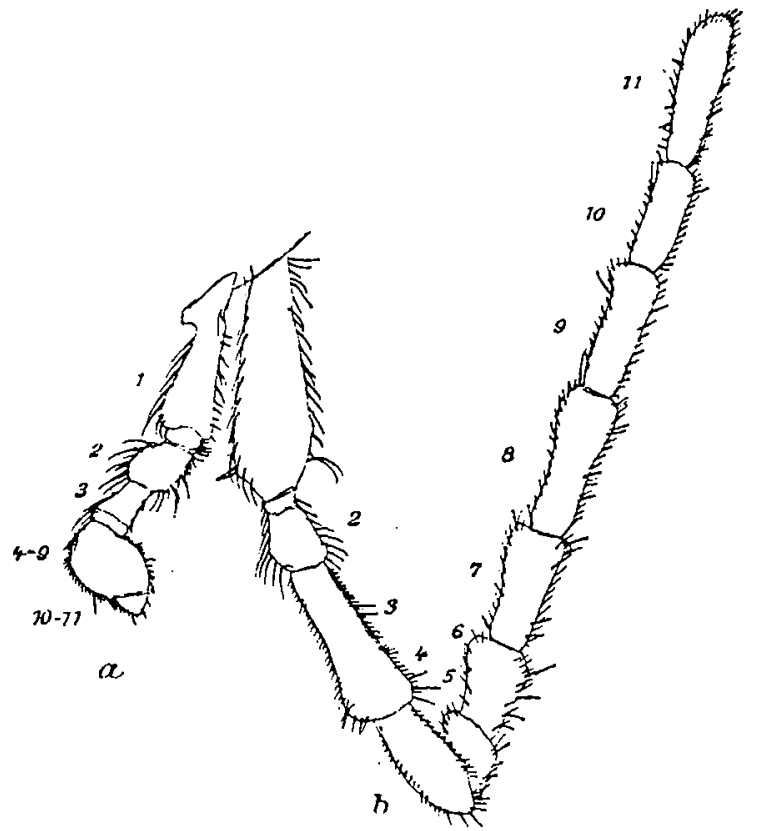

Abb. 1. Telephorus rusticus Fallen. Beide Fuhler ron links; $a$ Regenerat, $b$ normal (gezeichnet Zeiß-Komp. Ok. 6,0 bj. $a_{3}$, eingeschob. Tubus, dann fur Reproduktion auf $1 / 2$ verkleinert).

am Ende als dem normalen 8. (langes Haar) und 9. (kürzeres, starkes Haar) homolog darstellen, die Endkuppe also dem 10. plus 11. zu entsprechen haben; das 4. bis 9. Glied wären noch nicht zur Abgliederung gelangt, sondern stäken alle in der rundlichen Erweiterung.

Außer dem linken Fühler ist der Käfer normal ausgebildet.

Nr. 2. Telephorus violaceus Payk. (Textabb. 2, Taf. IV, Abb. 2). Rechter Fühler bis einschließlich des 3. Gliedes normal; an dieses schließen sich aber nur mehr zwei nach hinten gekrümmte Glieder, während der normale linke Fühler im ganzen 11 Glieder aufweist. Die zwei abnormen Glieder haben die typische Behaarung von Fühlerhgliedern, insbesondere stehen auf dem distaleren die drei sehr langen für das normale 11. Endglied charakteristischen Borsten. Der Gestalt nach ist das abnorme 
rechte Endglied dicker und kürzer, aber sonst dem normalen Endgliede im Fehlen geradliniger Begrenzungsstrecken ähnlich; das vorletzte Glied des abnormen Fühlers wäre also mit dem 4 . bis 10 . des normalen zu identifizieren. Mit Ausnahme der Krümmung und etwas größeren Ge-

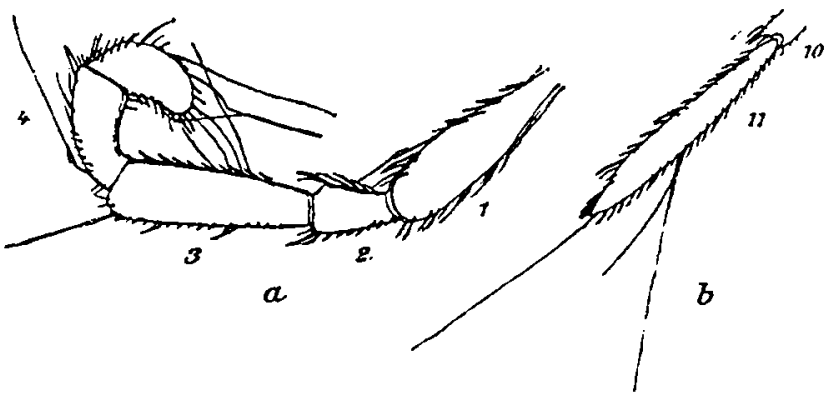

Abb. 2. Telsphorus violacsus Payk: Beide Fuhler von oben; a Regenerat des rechton, b normales Endglied des linken Fuhlers (gezeichnet wio Textabb. 1).

drungenheit weicht es nicht vom Aussehen eines Fühlermittelgliedes $a b$, insbesondere sind an ihm lange Sinneshaare zu erkennen.

Außer der abnormen Verkürzung des rechten Fühlers ist noch an der Unterseite des rechten Thoraxrandes eine Verletzung zu erkennen, welche in der Zerreißung der zum Vorderbeine führenden Chitinleisten besteht.

\section{Ersatzheteromorphose am Käferfühler.}

Nr. 3. Strangalia quadrifasciata L., (Textabb. 3, Taf. IV, Abb. 3). Der linke Fühler dieses Bockkäfers ist einschließlich des 6. Gliedes normal; auf dieses folgt ein nach vor- und abwärts gerichteter gegliederter Stummel, welcher die restlichen 5 Glieder in sich schließt. Diese Glieder entbehren durchaus der normalen Fühlerform und Behaarung und machen schon infolge ihrer Abbiegung von der Richtung der früheren Glieder nicht den Eindruck, als ob sie eine Fortsetzung des normalen Fühlers wären. Im einzelnen lassen sich folgende Charaktere feststellen: das 7. Glied ist erst wenig gedreht, wie sich aus der Oberansicht ergibt, trägt am Hinterrande eine Reihe längerer Borsten und weitet sich, wie in der Hinteransicht erkennbar, zu einer Manschette aus, die das 8. Glied empfängt. Aus dem kurz tütenförmigen 7. Glied biegt sich das walzenförmige 8. Glied nach vorn und unten $a b$; es trägt an der vorderen Seite einige längere Haare. In derselben Richtung schließt sich das 9. unbehaarte kleine Glied an. Das wieder schiof nach vorn und unten vorgezogene 10. Glied bildet eine lange Endfläche, aus deren oberem Ende das 11. Glied klauenartig schief gegen vorn und unten vorragt. 
Diese letzten zweiGlieder haben keine deutliche Behaarung, doch sind die Ränder rauh, als ob sie zur Borstenbildung schreiten wollten.

Außer dem linken Fühler war auch das linke Vorderbein verstümmelt worden, da es eine einfache runde Vernarbung im abgebrochenen Knie aufweist.
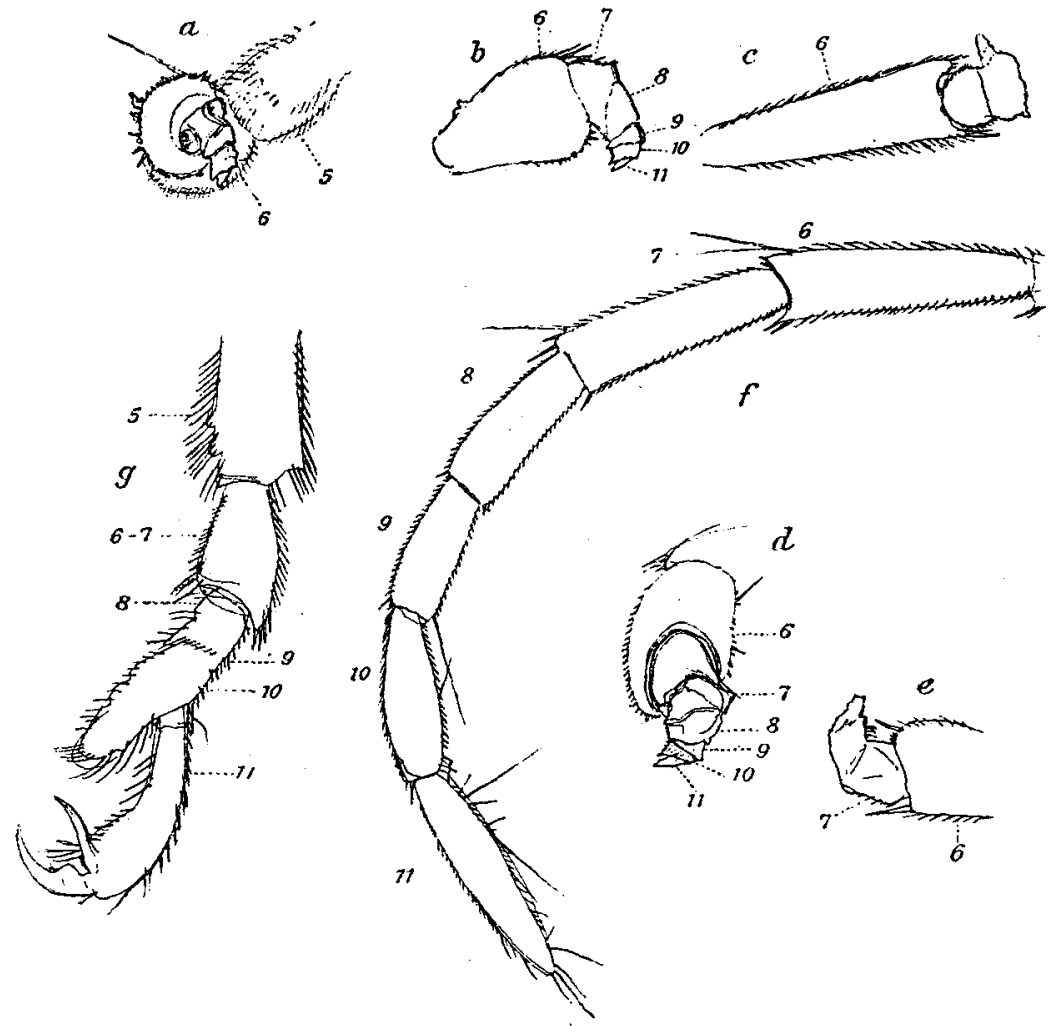

Abb. 3. Strangalia quadrijasciata L. a bis $e$ linke, heteromorphe Fühlerspitze, und zwar is von der linken Seite außen, $b$ von der linken Seite oben, $c$ von unten, $d$ von. hinten, i von oben gesehen; $f$ normale rechte Fühlerspitze; $g$ rechter linker VorderfuB von oben gesehen zum Vergleich mit $a$ bis $e$ (Vergrößerung wie Textabb. 1 und 2).

Im Hinblick auf die bei anderen Insektenordnungen bekannt gewordenen Ersatzheteromerphosen eines Fühlers durch ein Bein (vgl. die Literatur Przibram 1917 und 1918) habe ich untersucht; ob nicht der beschriebene Bockkäfer Merkmale von Fußgliedern an seinem abnormen Fühler aufweist. Die umstehende Tabelle bringt das Resultat.

Es zeigt sich, daß die richtige Zahl von Fühlergliedern angelegt ist, daß aber jedes der abnormen Glieder Ähnlichkeit mit einem der normalen Beinglieder aufweist, und zwar betreffen diese Ähnlichkeiten bloß die Fußglieder. Rechnen wir die Beinglieder analog den Fühler- 


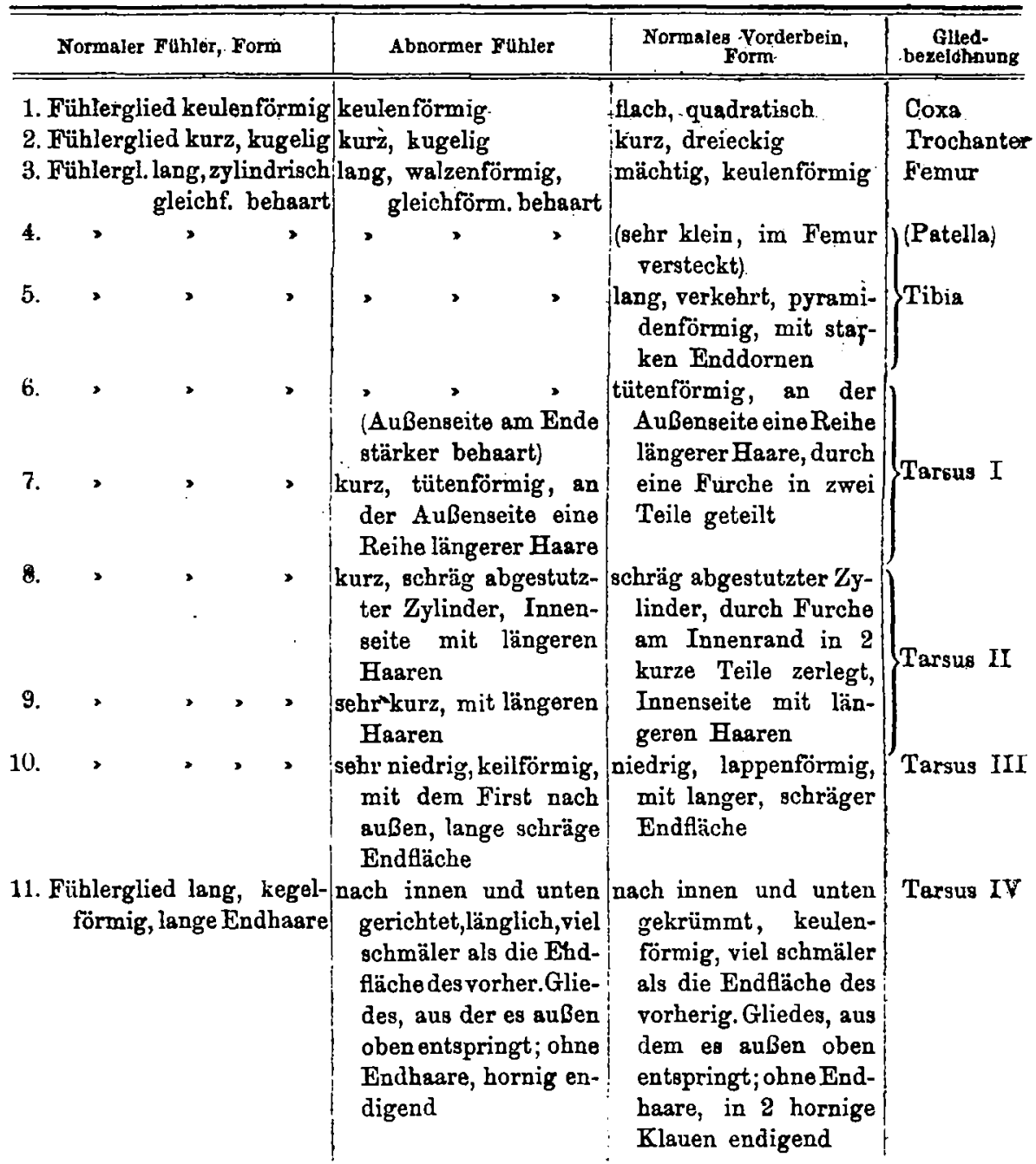

gliedern vom Körper an, so entsprechen die ersten fünf Beinglieder, welche alles bis zum Fußanfang enthalten, noch dem normalen Teile des abnormen Fühlers. Es war mithin auch nicht zu erwarten, daß andere Beincharaktere als $\mathrm{Fußmerkmale} \mathrm{auftreten} \mathrm{würden.} \mathrm{DaB} \mathrm{aber}$ auch andere Beinteile bei proximalerem Verluste mitauftreten könnten, haben mich die Experimente an Mantiden (1918) gelehrt. Nun besitzt aber der Fuß bei Strangalia, die zu den viergliedrigen Käfern gehört, nicht genug Glieder, als daß jedem der angelegten Fühlerglieder ein bestimmtes, anderes Fußglied entsprechen könnte. Das letzte and 
vorletzte Fußglied besitzen eine sehr ausgesprochene Form und gegenseitige Angliederung, und da wir diese letztere am abnormen Fühler wiederfinden, so habe ich Tarsalglied IV und III mit Fühlerglied 11 und 10 homologisiert. Es bleiben dann noch das I. und II. Tarsalglied übrig, welche nach der Stellung der Haare mit dem 6. plus 7., bzw. 8. plus 9. homologisiert werden können, indem die erstere Gruppe an der Außenseite, die letztere an der Innenseite die stärkeren bzw. längeren Haare aufweist. Unser Bockkäfer trägt also Merkmale aller Fußglieder an dem abnormen Fühlerstücke und wir können daher den Fall als Auftreten fußähnlicher Glieder an einem Fühler und demnach als iErsatzheteromorphose ". (Terminüs vgl. Przibram 1910) klassifizieren, da diese homoeotisch veränderten Fühlerglieder an der Stelle der normalen stehen. In den folgenden zwei Fällen handelt es sich hingegen um überzählige Zusatzglieder neben den normalen, also um "Zusatzheteromorphose «.

\section{Zusatzheteromorphose am Käferfühler.}

Nr. 4. Telephorus fuscus L. (Textabb. 4, Taf. IV, Abb. 4). Schneekäfer mit verzweigtem rechten Fühler. Derselbe ist bis zum 2. Gliede
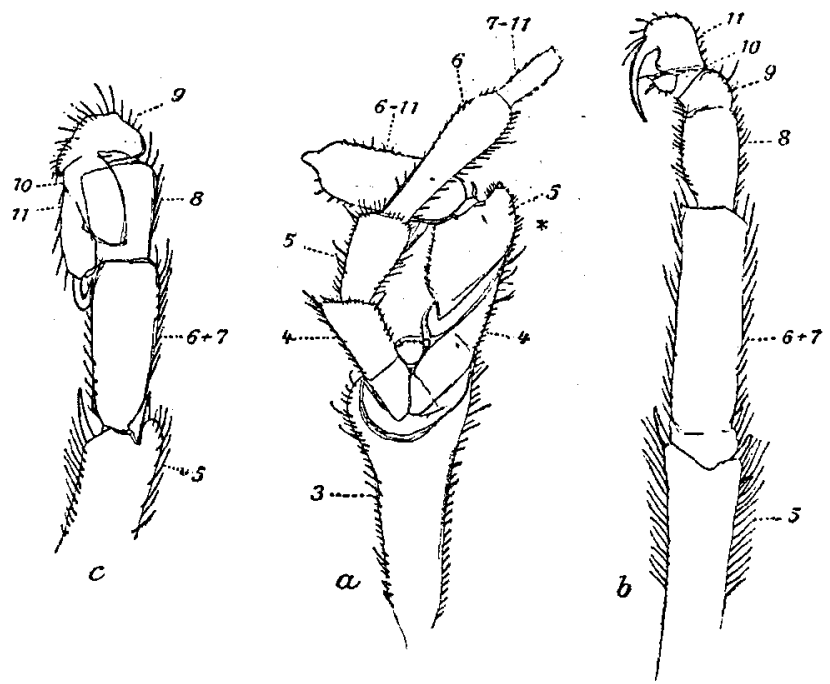

Abb. 4. Telephorus fascus L. a rechter Fühler mit überzähligem, heteromorphen Ast $(*)$ von unten, $b$ linker VorderfuB von oben, $c$ von unten zum Vergleiche (Vergrößerung wie Textabb. 1-3).

normal ausgebildet; das 3. Glied ist am Ende abnorm keulenförmig aufgetrieben und trägt zwei unter $60^{\circ}$ zusammenstoßende Gelenkfazetten, deren jede einer Reihe von Gliedern den Ursprung gibt. Die 
mehr nach hinten und unten gelegene Reihe besteht aus vier Gliedern, welche die typische Gestalt, Behaarung und Farbe der den normalen Fühler fortsetzenden Glieder 4 bis 7 bis auf die mangelhafte, wohl durch abermaligen Verlust bedingte Ausbildung des 7. Gliedes aufweisen. Der mehr nach vorn und oben liegende Fïhlerast besteht aus 3 Gliedern, welche von der Beschaffenheit normaler Fühlerglieder wesentlich abweichen. Zur Vermeidung von Weitläufigkeiten füge ich gleich die Tabelle mit der Homologisierung zu Beingliedern an.

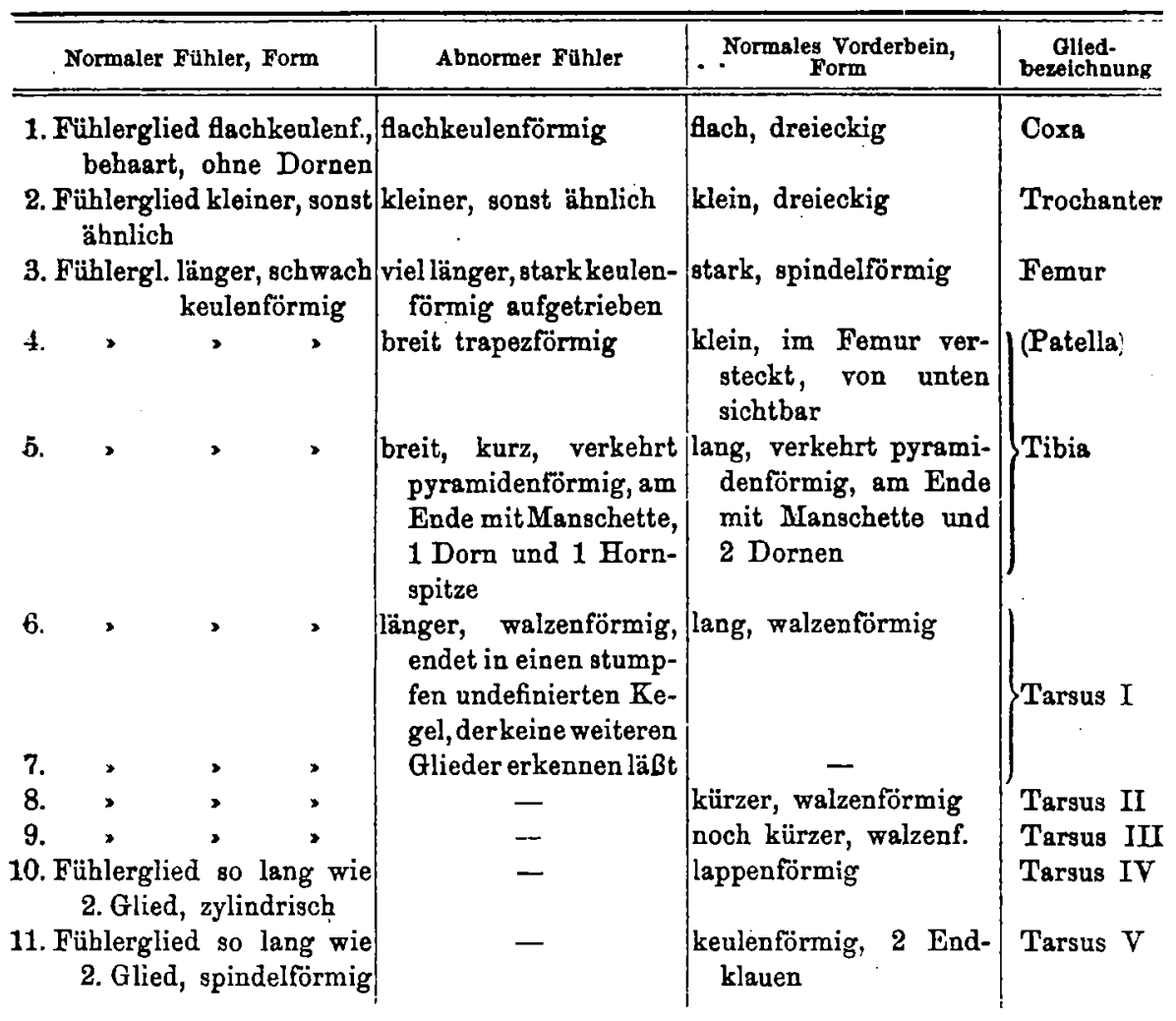

Da Hornspitzen und Dornen am normalen Fühler nirgends vorkommen, hingegen für das Ende der Tibia ebenso wie die manschettenartige Randvorwölbung charakteristisch sind, so läßt sich das 5. Glied des abnormen Fühlers mit einer stark verkürzten und auch sonst noch rudimentären Tibia vergleichen. Es wird vielleicht auffallen, daB dann das abnorme 4. Glied, welches an Mächtigkeit dem 5. fast gleichkommt, der im normalen Beine kaum sichtbaren Patella gleichgesetzt werden müBte. Doch habe ich bei Regeneraten gerade dieses Glied stets am 
deutlichsten gesehen und es kann der Schiene auf frühen Stadien der Wiedererzeugung gleichgro $B$ sein (vgl. Przibram).

Infolge der undeutlichen, wahrscheinlich auf Verlust zurückzuführenden Ausbildung des Endes läßt sich das noch vorhandene 6. Glied wohl als Fußglied, nicht aber gerade als ein bestimmtes mit Sicherheit bezeichnen. Ist das Ende nicht abermals abgebrochen, so hätten wir ein Sammelglied vor uns, das alle noch fehlenden einschließt, andernfalls kann es aber dem 6. Fühlerglied oder 1. Tarsalglied allein homolog sein.

Zu erwähnen ist noch, daß die Färbung der abnormen Glieder mehr jener der Beintibia und eines ersten Tarsalgliedes als den homologen normalen Fühlergliedern entspricht, indem der rotgelbe Bezirk bei letzteren durch Verdunkelung vom distalen Ende aus bereits fast ganz auf die proximalste Stelle beschränkt bleibt, während bei den beiden ersteren nur der Vorderrand und schmale Seifenränder geschwärzt erscheinen.

Nr. 5. Carabus festivus (Textabb. 5, Taf. IV, Abb. 5). Die rechte Antenne dieses Laufkäfers ist am Ende des 7. Fühlergliedes nach abwärts abgeknickt. Aus der Knickungsstelle erhebt sich ein dreigliedriges Zusatzgebilde, welches, dicker als das 8 . Fühlerglied beginnend, symmetrisch zu demselben nach aufwärts sieht. Das 1. Glied dieses Zusatzgebildes ist mit dem 8 . Gliede des abgebogenen ursprünglichen Fühlerastes zusammengestoßen und die Richtung der Dornen am Innenrande zeigt deutlich, wo dieses Gebilde die ursprüngliche Wachstumsrichtung des 7. Gliedes umgekehrt hat (auf der Textabb. mit einem Sternchen bezeichnet). Von dieser Stelle an läuft die Bedornung derjenigen des normalen 8. Gliedes entgegengesetzt und tritt die Ausstattung mit Sinneshaaren und Enddornen der Gegenseite, also eines linken Fühlers, auf. Das 2. Gilied des Zusatzes, ebenfalls dicker und kürzer als das ent. sprechende normale 9. Glied, zeigt noch typische Bedornung eines Fühlergliedes, ist auch mit Sinneshaaren ausgestattet. Die Symmetrie ist wenig klar. Aus der breit abgestutzten Endfazette entspringt ein langes schmales, spindelförmiges, vor dem Ende keulenartig sich erweiterndes Glied. Dasselbe entbehrt der Dornen, Borsten und Sinneshaare der normalen Fühlerglieder und endet'in einen rechteckigen, noch viel schmäleren Fortsatz, der abgesetzt, aber nicht abgegliedert ist und an der einen Seite ein klauenförmig gekrümmtes Anhängsel, auf der anderen ein wenig vorragendes Knöpfchen trägt. Ventral zieht am Endgliede der Länge nach eine Leiste, welche von Ansätzen zu Sägezähnen begleitet wird. Dieses Glied hat keine Ähnlichkeit mit einem Fühlergliede, am wenigsten mit den beiden letzten, denen es der Reihenfolge nach entsprechen müßte. Hingegen läßt sich die Ähnlichkeit mit dem letzten Tarsalgliede (z. B. des abgebildeten Vorderbeines) nicht verkennen. Auch dieses ist viel schmäler als das vorhergehende Glied, lang, spindel- 
förmig, vor dem Ende keulenförmig verdickt, endet in eine rechteckige Sohlenplatte und zwei Krallen, die freilich mächtig ausgebildet: sind. Es hat fast glatte Konturen; keine feine Bedornung oder Behaarung;

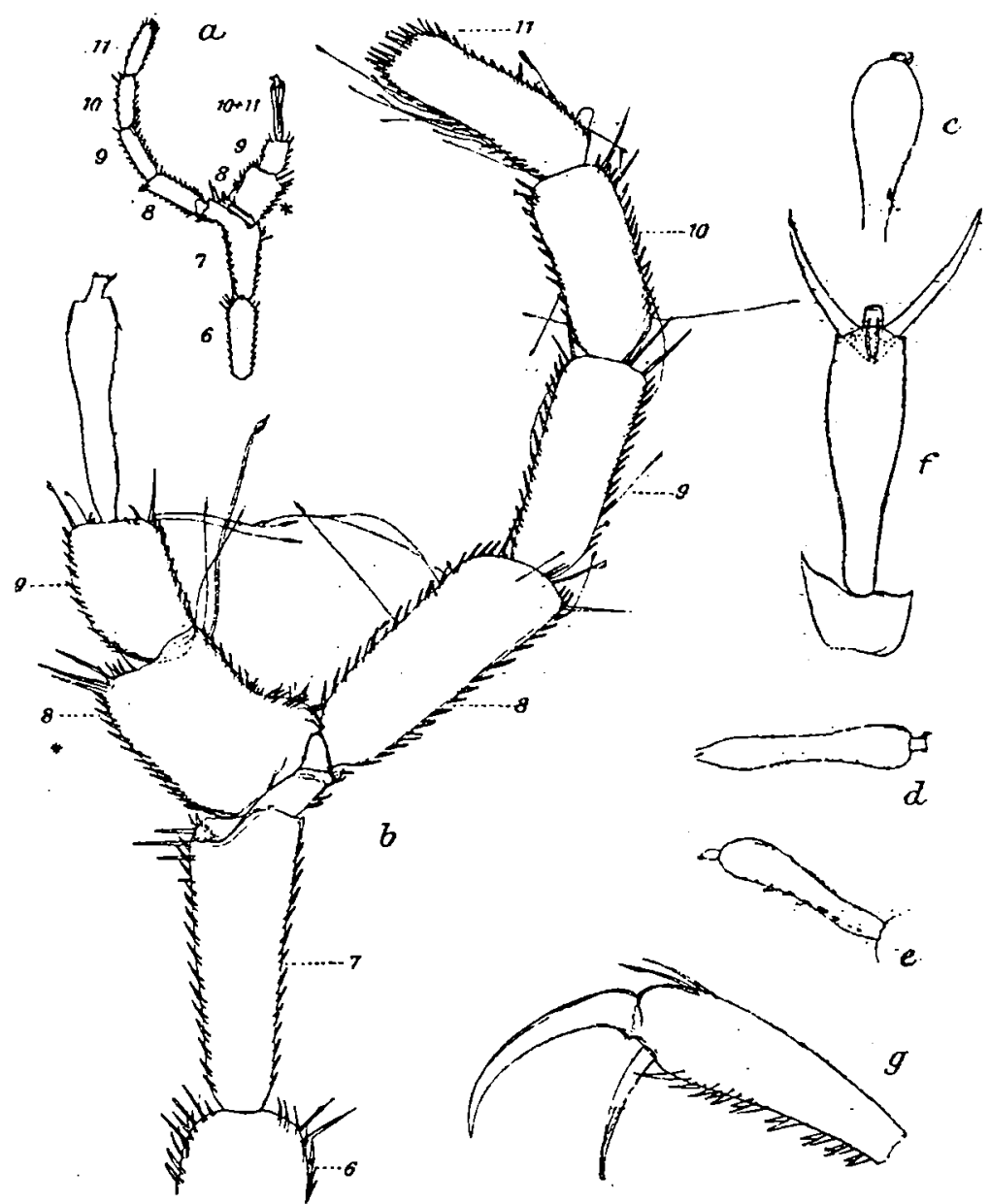

Abb. б. Carabus festivus $\mathrm{L}$. a rechter Fuhler mit uberzahligem, heteromorphem Aste (*) von der rechten Seite (LupenvergröBerung fur die Roproduktion auf $1 / 2$ verkleinert); $b$ derBelbe von der linken Seite; $c$ Endigung von oben, $d$ von außen, $e$ von auBen-unten; $f$ letzte Tarsalglieder von oben zum Vergleiche mit $c ; g$ letztes Tarsalglied des linken - Vorderbelnes von auBen-unten gesehen zum Vergleiche mit $e$ ( $b$ bls $g$ VergröBerung wio Textabb. 1-4).

auf der Unterseite sind aber zwei Reihen starker Kammzähne vorhainden, welche die auch beim abnormen Gliede erwähnte Leiste begleiten und oberseits vor dem Ende zwei Tastborsten, die dem abnormen Zusatz: gliede fehlen. 


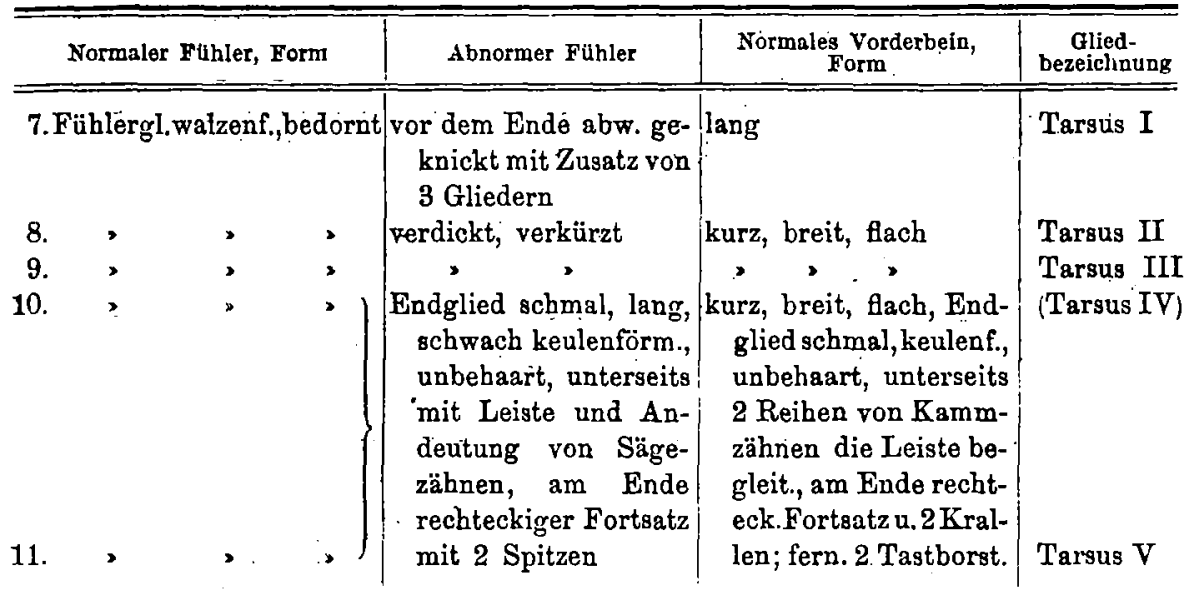

\section{Theoretische Erörterung.}

Für die theoretische Deutung der fußähnlichen Glieder an Fühlern ist es zunächst wichtig nachzuweisen, daß wir es wirklich in den beschriebenen Fällen mit Regeneraten zu tun haben. Eine tabellarische Ubersicht der" 5 beschriebenen Käfer wird eine weitschweifige Wiederholung bereits im einzelnen vorgebrachter Indizien vermeiden lassen.

Tabelle über die 5 Käfer mit abnormem Fühler.

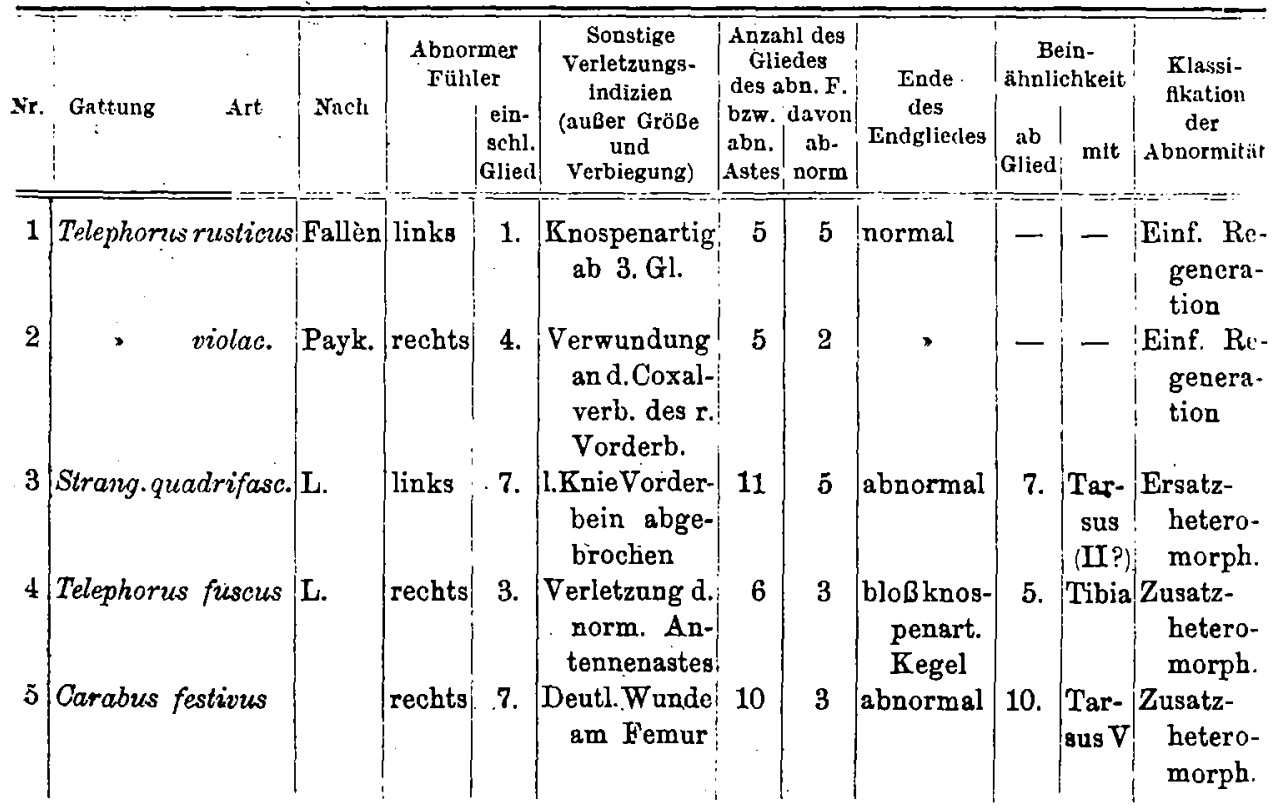


In allen Fällen finden sich in der Umgebung des abnormen Fühlers noch andere Verletzungsspuren als die typischen Verbiegungen der betroffenen Fühlerteile und die geringere Länge und Ausbildung mit Ausnahme von $\mathrm{Nr}$. 1, wo aber gerade die durchweg geringere Größe der Glieder und die unvollständige Abgliederung für Regeneration spricht.

Man könnte nun glauben, daß die beschriebenen fußähnlichen Fühlerglieder nur weniger gut ausgebildete Fühlerglieder seien, welche erst die typische Form, Bewehrung und Behaarung erlangen sollten, woran sie freilich durch den Eintritt des Imaginalstadiums gehindert worden wären.

Wäre diese Interpretation richtig, so müßten wir erwarten, die normalste Ausbildung der Fühlerglieder bei jenen Abnormitäten anzutreffen, welche der normalen Gliederanzahl bei der fortschreitenden Regeneration am nächsten gekommen sind. Das ist aber nicht der Fall: gerade jene beiden Nummern 1 und 2, welche normaler Ausbildung der bereits vorhandenen Fühlerglieder nahekommen, sind am weitesten von der Erreichung der normalen Fühlergliedzahl entfernt.

Insbesondere hat das Endglied an diesen Regeneraten die typische Endbehaarung des 11. Fühlergliedes, was der allgemein für die Arthropodenfühler gültigen Regel entspricht, daß zuerst das Spitzenglied zur: Regeneration gelangt (Przibram 1899, Zelen y 1907, Hase man 1907).

Bei den fußähnlichen Fühlern finden wir nicht diese zuerst beim Regenerate zu erwartende Spitzenausbildung. Obzwar bei Nr. 3 alle verloren gewesenen Fühlerglieder der Zahl nach richtig wiederaufgetreten sind, ist doch keine typische Fühlerendigung vorhanden; bei Nr. 5. die fast die normale Fühlerzahl erreicht, fehlt ebenfalls diese Endigung, und Nr.4, bei der die Abnormität deutlich vom 3. Gliede ausgeht und 3 weitere Glieder gut ausgebildet hat, ist auch die Spitze erst als Knospe ohne Endborsten angelegt.

Wir könnten sogar diese zentrifugale Abgliederungsrichtung als ein weiteres zugunsten der Fußähnlichkeit sprechendes Merkmal in Anspruch nehmen, wenn es sich bestätigen sollte, daB diese Abgliederungsrichtung auch für die normale Regeneration von Beinen bei Käfern gilt, wie sie für andere Arthropoden von Haseman (1907) im Gegensatze zu der anfangs zentripetalen Abgliederungsrichtung an den Antennen beobachtet worden ist.

Damit wäre auch die geringe Andeutung der Fußklauen vereinbar, welche die fußähnlichen Fühler aufweisen. Doch läßt sich diese Frage ohne speziell hierauf gerichtete Versuche nicht entscheiden.

Warum ich nicht zweifle, die fraglichen Fühler als fußähnlich zu bezeichnen, das ist schließlich das Vorkommen von deutlich in Füße endi. genden Fühlern bei anderen Insektenordnungen, z. B. Hymenopteren (darunter ein Zusatz) und Orthopteren (vgl. Przibram 1917, III). 
Neuerdings konnte ich bei Sphodromantis die Umwandlung des gänzlich entfernten Füblers in ein fast vollständiges Bein erzwingen (Przibram 1918) und ähnliches wird Leonore Brecher demnächst von Stabheuschrecken zu berichten haben.

Können wir das Auftreten von Fußcharakteren an Käferfühlern als Heteromorphosen auffassen; so ergeben sich eine Reihe von Schlußfolgerungen in bezug auf den Einfluß des Nervensystems auf die Regeneration, welche den bisher adoptierten Anschauungen widersprechen.

Der von Herbst (1895-1899) geführte Nachweis, daß die an Stelle von Augen auftretenden fühlerähnlichen Gebilde bei Krustern an die Entfernung des augengangliontragenden Stieles gebunden sind und namentlich auch die neben Augenteilen auftretenden Antennengeißeln (Howes 1887) durch Auskratzung des Ganglions unter Erhaltung des Stieles nachgeahmt werden können (Herbst 1901), haben nicht nur diesen Autor, sondern auch die meisten übrigen Forscher von dem "formativen " Einflusse des Ganglions überzeugt, das als - "formativer Reiz \& auf das Regenerat einwirken sollte. Ich war selbst geneigt, einen derartigen Einflu $B$ des Nervenzentrums auf die entstehenden Regenerate von Arthropodenanhängen im allgemeinen anzunehmen, daß die Entfernung des zugehörigen Ganglions die normale Ausbildungshöhe des Anhanges erlöschen macht, so daß nur ein niedrigerer Zustand zur Ausbildung gelangt (Przibram 1910). Dabei bin ich aber auf technische und sachliche Schwierigkeiten gestoßen, namentlich bei dem Ersatze von Insektenfühlerspitzen durch Füße, wo von einer Entfernung des Fühlerganglions keine Rede sein konnte, während die in der Antennengeißel vorfindlichen sog. "Ganglien «fraglicher Natur (vgl. Przibram 1917, S. $82 \mathrm{Anm}$.) acuh in den Geißeln der Krebse sich vorfinden, ohne daß bei Abschnitt der Antenne ihre Regeneration als solche ausbleiben würde. Trotzdem habe ich bisher, wenn auch unter Betonung der Unsicherheit, an der Hypothese festgehalten, solange kein zwingender Gegenbeweis vorlag. Ubrigens regenerieren die sog. "Ganglien " der Insektengeißel mit. In letzter Zeit ist Herbst (1916, S. 472) bezüglich der Notwendigkeit des Ganglions für die richtige Ausformung der regenerierenden Krebsanhänge durch Versuche an Schwanzbeinen von Palaemon schwankend geworden, da hier nach Ganglienexstirpation Schwanzbeine als solche nachwachsen, und zwar ohne mitfolgender Wiederherstellung des Ganglions.

Giesebrecht (1910), welcher die Abdominalbeine nach Ganglienexstirpation unverändert regenerieren sah, hatte vorgeschlagen, zwischen Sinnesorganen und Bewegungsorganen zu unterscheiden; nur bei ersteren sollen die nervösen Zentren einen formativen Einfluß ausüben, nicht aber bei letzteren. Gegen diese Trennung hatte Herbst insofern mit Recht Bedenken, als Giesebrecht die regenerierenden heteromorphen 
Antennulen nun nicht durch formativen Einfluß der Ganglien gleich den Augen, sondern als Bewegungsorgane ohne solchen Einflu $B$ sich entstehen dachte. Auch nach Giesebrechts Anschauung müßte also gleichwie Herbst es sich vorgestellt hatte, ein formativer Einfluß des Ganglions der benach barten 1. Antenne auf die Heteromorphose bestehen.

Wollten wir aber diese Annahme auf andere homoeotische Regenerationen ausdehnen, so müßten die ersetzenden und ersetzten Anhänge stets benachbarten Segmenten angehören oder zumindest in den Ganglien durch direkte Kommissuren verbunden sein, denn es ist schwer einzusehen, wie der "formative " Einfluß der weiterliegenden Ganglien über die unverletzten dazwischenliegenden hinübergreifen solltè.

Nun ist dies aber bei der Ersetzung des Fühlerendes durch FuBglieder, wenngleich es sich wahrscheinlich stets dabei um das Vorderbein handelt, nicht der Fall, da dem Fühlersegmente keineswegs das 1. Thorakalsegment folgt, dem das l. Beinpaar angehört, vielmehr schieben sich die Mundwerkzeuge ein.

Jedenfalls würden auch die gleichartigen Ganglien der Gegenseite näher liegen: Aber es tritt nun bei unseren Käfern eine weitere Schwierigkeit auf, die selbst die Deutung durch Kommissuren unmöglich zu machen scheint.

Bei den Zusatzheteromorphosen von fußähnlichen Gliedern an Fühlern fällt die Entstehungszeit selbstredend in die larvale Periode, denn nur in dieser können verlorene oder verwundete Teile nach wachsen (wollte man den Regenerationscharakter nicht anerkennen, so würde die Entstehung in eine noch frühere Embryonalperiode verlegt werden müssen). Nun muß doch der verletzte larvale Fühler sich in den Imaginalfühler verwandelt haben, desgleichen sein abnormer Ast. Während nun der normale Fühler bei dieser durchgreifenden Metamorphose unter dem formativen Einfluß des Fühlerganglions gestanden sein muß müßte nur der abnorme Ast allein sich teilweise demselben entzogen und unter den Einflu $\beta$ des Vorderbeinganglions geraten sein und beide formative Einflüsse würden durch die Nerven des unversehrten Fühleranfanges vermittelt worden sein! Wollte man, wie Herbst provisorisch für die Abdominalbeine diskutiert, eine "Nachwirkung" des entfernten oder verletzten Ganglions annehmen, so muß man sich fragen, warum diese "Nachwirkung « sich nicht auch bei Auskratzung des Augenstieles bemerkbar macht, um ein Auge zu ergänzen?

Ich glaube, daß alle diese Annahmen an unsere Gläubigkeit bereits eine zu große Anforderung stellen und es daher besser ist die Hypothese des formativen Ganglieneinflusses auf der Nervenleitungsbahn fallen zu. lassen, welche ohnehin bloß für die Arthropoden zu gelten schien. An dieser Stelle möchte ich mich nicht weiter über die Frage des Nerveneinflusses auf die Regeneration anderer Gruppen einlassen. Es genügt 
an die Regenerationsfähigkeit eines an beliebige Körperstelle transplantierten Triton-Beines (Kurz 1912) und an die Wiederherstellung der Augenelemente an ebenso verpflanzten Salamanderaugen (Uhlenh u th) zu erinnern, um für die Wirbeltiere jede Notwendigkeit zugehöriger Ganglien für die Ausformung in Abrede zu stellen.

Neuerdings hat Kopeć (1917) einen analogen Versuch mit Verpflanzung von Raupenaugen auf ein Abdominalsegment gemacht und die normale Verwandlung zum Imaginalauge bei Lymantria dispar beobachtet. Derselbe Autor konnte verschiedene Ganglien an den Raupen entfernen, ohne daß die von ihnen innervierten Gebiete an ihrer normalen Entwicklung und Metamorphose verhindert wurden. Selbst nach Entfernung des Gehirns verwandelte sich das Auge normal, und bei Abwesenheit zentraler Ganglien und Fußnerven regenerierten dennoch die abgeschnittenen Abdominalfüße an den Raupen.

Die unabhängige Entwicklung der Transplantate gibt uns den Anhaltspunkt, daß die formativen Potenzen im Organ selbst gelegen sind, nicht von einem nervösen Ganglion auf dem Wege der Nervenbahn ausgeübt werden. Die Deutung, welche Herbst seinerzeit als zweite Alternative aufgestellt, aber nicht adoptiert hatte, daß zwei Potenzen in den zur Heteromorphose befähigten Gliedmaßen vorhanden seien, von denen die eine nach Erlöschen der anderen sich betätigt, liefert uns befriedigendere Erklärung.

Wir können noch weitere Tatsachen anführen, welche für diese Deutung sprechen: a) die meistens geringere sinnesphysiologische Differenzierungshöhe des ersetzenden gegenüber dem ersetzten Organe, welche auf das Ubrigbleiben einer allgemeineren, weniger differenzierten Potenz verweist (vgl. die Reihe der Homoeosis - Przibram 1910 und die teilweise Korrektur). Würde es sich um Einfluß benachbarter Nerven handeln, so hätte man eher an eine stärkere Beeinflussung durch die sinnesphysiologisch höher stehenden Organe im Sinne Giesebrechts denken können.

b) Bei den im Systeme niedriger stehenden dekapoden Crustaceen bleibt die Potenz zur Fühlerbildung noch nach derselben Operation bestehen (vgl. Przibram 1901), nach der bei den Hexapoden (vgl. Przibram 1917, 1918) Heteromorphose des Fühlers eintreten kann, in Ubereinstimmung mit der Regel, daß höhere Formen früher der vollständigen Regeneration verlustig gehen, als niedrigere.

c) Bei Versuchen an Sphodromantis wurden die am meisten beinähnlichen Fühlerersätze nach Operation an alten Stadien erreicht, während jüngere Larven fast normalen Fühlerzustand zu erreichen imstande waren. Dies stimmt zu einer Abnahme der höherdifferenzierten Potenz mit dem Alter. Bei einer "Nachwirkung « des Nerveneinflusses müßte man eher das Umgekehrte erwarten, da die Abhängig- 
keit der Funktionen vom Nervensystem mit der Entwicklung zunimmt, was Roux (1905) sogar zur Aufstellung von zwei Perioden, einer mehr unabhängigen embryonalen Entwicklung und einer späteren funktionellen' Abhängigkeit bewog.

\section{Zusammenfassung.}

Die Beschreibung einiger im entwicklungsmechanischen Museum der Anstalt befindlichen, im Freien gesammelter Käfer mit Fühlermonstrositäten gibt zu folgenden Erörterungen Anlaß:

I. An normalen Regeneraten des Käferfühlers (Nr. 1. Telephorus rusticus und Nr. 2. T. violaceus) fand sich wie bei den übrigen bisher untersuchten Arthropoden das Spitzenglied mit seiner charakteristischen Behaarung angelegt, ehe noch die normale Gliederzahl erreicht war.

II. Das an einem Käferfühler (Nr. 3. Strangalia quadrifasciata) aufgetretene vollgliedrige Regenerat, welches keine typische Ausbildung des Fühlerendes aufweist, vielmehr fußähnliche Glieder trägt, wird daher nicht als noch wenig ausgebildetes normales, sondern als heteromorphes Regenerat angesprochen, und zwar als "Ersatzheteromorphose ".

III. Zwei weitere Käferfühler (Nr. 4. Telephorus fuscus und $\mathrm{Nr} .5$ Carabus festivus) tragen neben dem abgebogenen, normalen Fühlerende einen abnormen Ast, dér Fußcharaktere aufweist, und werden als "Zusatzheteromorphosen " betrachtet im Gegensatze zu den meisten bisher bekannten überzähligen Fühlerästen bei Käfern mit normaler Endauعbildung.

IV. Da die erwähnten Monstrositäten sich als Regenerate zu erkennen geben, welche auf Verletzung im Larvenzsutande zurückzuführen sind, und die beiden Äste die Metamorphose durchgemacht haben, so müßte die Annahme der Imaginalgestalt beim normalendigenden Aste unter dem Einflusse des Fühlerganglions, beim abnormalen aber unter dem Einflusse eines Beinganglions gestanden sein, wenn man einen formativen EinfluB der Ganglien auf dem Wege der Nervenbahn (Herbsts erste Alternative) annehmen wollte.

V. Da der Einfluß des Beinganglions aus einem nicht unmittelbar an das Fühlersegment grenzenden Körperabschnitte durch den normalen und daher wohl auch normal innervierten noch einfachen Teil des Fühlers hindurch wirken müBte, um zum heteromorphen Ast zu gelangen, so wird die Nervenbeeinflussung abgelehnt und eine zwiefache Potenz jedes Arthropodenanhanges (Herbsts zweite Alternative) angenommen.

VI. Es zeigt sich, daß diese Hypothese auch mit den übrigen über Regeneration und Transplantation bekannten Tatsachen bei Vertebraten und Arthropoden besser harmoniert als die frühere. 


\section{Literaturverzeichnis.}

Gadeau de Kerville, H., Expériences tératogéniques sur differentes espèces d'insectes. Le Naturaliste. 115.

1890.

Giesebrecht, W., Monographie: Stomatopoden, in: Fauna u. Flora d. Golfes von Neapel. XXXIII. 203.

1910.

Haseman, J. D., The Direction of Differentiation in Regenerating Crustacean Appendages. Arch. f. Entw.-Mech. XXIV. 617.

1907.

- The Reversal of the Direction of Differentiation in the Chelipeds of the Hermit Crab. Arch. f. Entw.-Mech. XXIV. 663.

1907.

Herbst, C., Über die Regeneration von antennenähnlichen Organen an stelle von Augen. I. Mitteilung. Arch. f. Entw.-Mech. II. 544. 1895- 1896.

-- II. Mitteilung. Versuche mit Sicyonia sculpta. Vierteljahrsschrift muturforschender Gesellschaft Zürich. XLI. 435 (Jubelband). 1896.

- III. Weitere Versuche mit total exstirpierten Augen und IV. Versuch ${ }^{*}$ mit teilweise abgeschnittenen Augen. Arch. f. Entw.-Mech. IX. 215. 1899.

- V. Weitere Beweise für die Abhängigkeit der Qualität des Regemerates von den nervösen Zentralorganen. Arch. f. Entw.-Mech. XIII. 4:36. 1901.

- VI. Die Bewegungsreaktionen, welche durch Reizung der heteromorphen Antennuli ausgelöst werden. Arch. f. Entw.-Mech. XXX. 1. (RouxFestschrift).

1910.

- VIr. Die Anatomie der Gehimnerven und des Gehirnes bei Krebsen mit Antennulis an Stelle von Augen. Arch. f. Fntw.-Mech. XLII. 407.1916.

- Uber die Regeneration der Schwanzbeine von Palaemon nach Fntfernumy der Schwanzganglien. Arch. f. Entw.-HIech. XIIII. 329.

Howes, G. B., Exhibition and remarks upon an original drawing of the heiul of an abnormal Palinurus. Proceedings Zoological Society Lonclon. 468.

1887.

K opec, S., Experiments on Metamorphosis of Insects. Bulletin Acaclémie Cracovie: Janvier-Mars. 57.

$191 \%$.

Kurz, O., Die beinbildenden Potenzen entwickelter 'Tritonen. Arch. f. Entw. Mech. XXXIV. $\mathbf{5 8 7 .}$

1912.

Przibram, H., Die Regeneration bei den Crustaceen. Arbeiten zoologischer Institute Wien. XI. 163.

1899.

- Experimentelle Studien äber Regeneration. Arch. f. Entw.-Mech. XI. 3:1.

-- Die Homocosis bei Arthropoden. Arch. f. Entw. -Mech. XXIX. 588.

-- Transitiire Scherenformen usw. (zugl. Exp. Stud. V. und Homoeosis V.). Ar(h. f. Entw.-Nech. XLIII. 47.

1917.

- Fühlerregeneration halberwachsener Sphodromantis-Larven (zugleich Aufaucht der Gottesanbeterinnen IX. und Homoeosis III.). Arch. f. Fintw.-A[ech. XIIII. 64.

1917.

- Fangbeine als Regenerate (zugl. Aufzucht IX. und Homoensis IV.). Arch. $t$. Entw.-Nech. XLV. 39.

1919.

Roux, W., Die Entwicklungsmechanik. Vorträge und Aufsïtze. Heft I. Jeeijurir. Engelmann. (S. 94.)

1905 .

Tornier, G., Das Entstehen von Käfermißbildungen, besonders der Hyperattennit und Hypermelie. Arch. f. Entw.-Mech. IX. 501. 1900.

- Bein- und Fühlerregeneration bei Käfern und ihre Begleiterscheinungen. Zoulo. gischer Anzeiger. XXIV. 634.

1901. 
Uhlenhuth, E., Die Transplantation des Amphibienauges. Arch. f. Entw.-Mech. XXIII. 723.

1912.

- Synchrone Metamorphose des transplantierten Salamanderauges. Arch. f. Entw.-Mech. XXXVI. 211.

1913.

Werber, J., Regeneration des exstirpierten Fühlers und Auges beim Mehlkäfer, Tenebrio molitor. Arch. f. Entw.-Mech. XIX. $259 . \quad 1904$.

Zeleny, Ch., The Direction of Differentiation in a Regenerating Appendage.

Science N. S. XXIII. 526.

1906.

- The Direction of Differentiation in development. 1. The antennulae of $\mathbf{M a n}$ casellus macrourus. Arch. f. Entw.-Mech. XXIII 324. 1907.

\section{Tafelerklärung. \\ Tafel IV.}

Abb. I. Telephorus rusticus Fallèn, Kopf und Halsschild. von oben; linker Fühler normales Regenerat. - Wien.

Abb. 2. T. violaceus Payk., Kopf und Halsschild von unten; rechter Fühler normales Regenerat. - Semmering.

Abb. 3. Strangalia quadrifasciata L., Kopf mit linkem, in fußähnliche Glieder endigenden Fühler, von oben. - Semmering.

Abb. 4. Telephorus fuscus L., Kopf und Halsschild von oben; rechter Fühler mit fußgliederähnlichem Aste. - Wien.

Abb. 5. Carabus festivus L., Kopf und Halsschild von oben; rechter Fühler mit fußgliederähnlichem Aste. - Mehadia (Ungarn). 


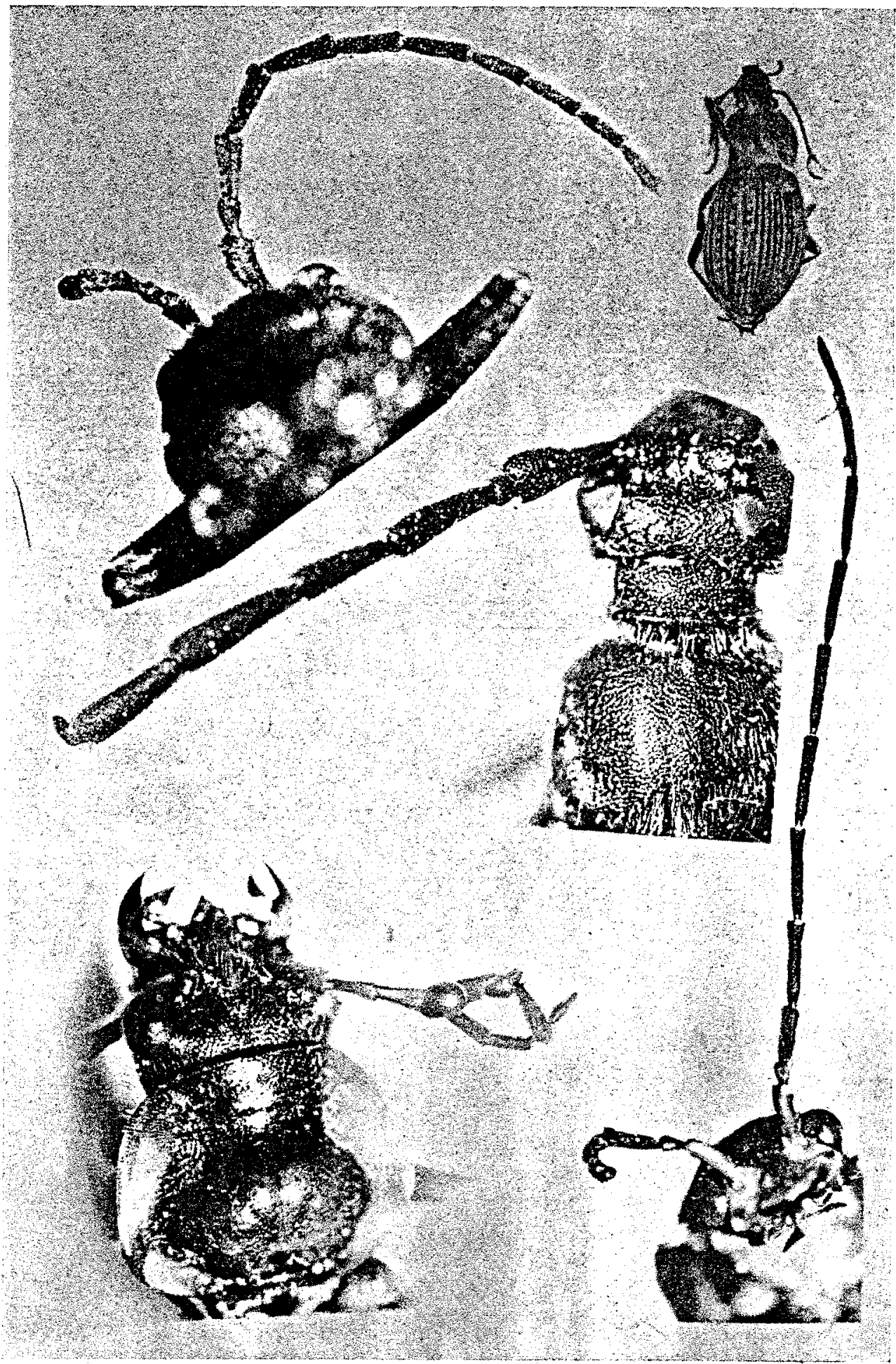

